\title{
3D lens-free time-lapse microscopy for 3D cell culture
}

\author{
Anthony Berdeu ${ }^{1,2}$, Fabien Momey ${ }^{3}$, Bastien Laperrousaz ${ }^{1,4,5}$, Thomas Bordy ${ }^{1,2}$, Xavier \\ Gidrol $^{1,4,5}$, Jean-Marc Dinten ${ }^{1,2}$, Nathalie Picollet-D’hahan ${ }^{1,4,5}$, Cédric Allier ${ }^{1,2}$ \\ ${ }^{1}$ Univ. Grenoble Alpes, 621 Avenue Centrale, 38400 Saint-Martin-d'Hères, France \\ ${ }^{2}$ CEA, LETI, MINATEC Campus, 17 Avenue des Martyrs, 38000 Grenoble, France \\ ${ }^{3}$ Laboratoire Hubert Curien - Univ. Jean Monnet, 18 Rue Professeur Benoît Lauras, 42000 Saint-Étienne, France \\ ${ }^{4}$ CEA, BIG, Biologie Grande Échelle, 17 Avenue des Martyrs, 38000 Grenoble, France \\ ${ }^{5}$ INSERM, U1038, 17 Avenue des Martyrs, 38000 Grenoble, France \\ anthony.berdeu@cea.fr,fabien.momey@univ-st-etienne.fr
}

\begin{abstract}
We propose a new imaging platform based on lens-free time-lapse microscopy for $3 D$ cell culture and its dedicated algorithm lying on a fully $3 D$ regularized inverse problem approach. First $3 D+t$ results are presented.
\end{abstract}

OCIS codes: (090.1995) Digital holography, (180.6900) Three-dimensional microscopy.

\section{Introduction}

The study of in-vitro cell populations is a challenge if one wants to gather quantitative and systematic data over extended periods of time on a large volume while preserving the integrity of the living sample. Lens-free time-lapse microscopy provides a simple, cheap and robust tool meeting these needs in the realm of 2D cell culture [1-3]. Along with the standardization of 3D culture protocols in cell biology, lens-free imaging techniques must also be extended to this new modality both in terms of acquisition methods and $3 \mathrm{D}$ reconstruction algorithms.

We present here a new 3D lens-free microscope for large 3D biological samples and a dedicated reconstruction algorithm based on the Fourier diffraction theorem as used in standard diffractive tomography [4-6]. It uses a regularized inverse problem formalism directly applied on the whole dataset and the 3D object to retrieve.

Reconstructed volumes as large as $\sim 5 \mathrm{~mm}^{3}$ are given with a resolution sufficient to resolve isolated single cells. First results of a 3D reconstruction along the time are also presented.

\section{Materials and methods}

Standard 2D lens-free microscopes are minimalist tools based on the in-line holography principle (see Fig. 1.a) proposed by Gabor in 1948 [7]. The 2D cell culture is placed on the top of the sensor at a distance of 1 to $3 \mathrm{~mm}$ and diffracts the normally incident light. The object is then numerically retrieved from the recorded hologram.

We developed an experimental bench to adapt this geometry for the reconstruction of $3 \mathrm{D}$ objects which requires to multiply the viewing angles (see Fig. 1.a). The object is static while the sensor and the multi-wavelength illumination source are rotating around an axis orthogonal to the sensor with a stepper motor. Unlike the 2D in-line geometry, the illumination is tilted by an angle $\theta=45^{\circ}$. This configuration is well adapted to standard culture cell containers such as Petri dishes.

The 3D diffracting sample is described by its scattering potential $f$ defined at each point of space $\vec{r}=(x, y, z)$ by:

$$
f(\vec{r})=\left(\left(n(\vec{r}) / n_{0}\right)^{2}-1\right)
$$

where $n(\vec{r})$ is the local refractive index and $n_{0}$ is the refractive index of the surrounding medium.

Using the notations introduced on Fig. 1.b, in the case of a monochromatic incident plane wave $U_{\text {inc }}(\vec{r})=e^{i \overrightarrow{k_{0}}} \cdot \vec{r}$ of wave vector $\overrightarrow{k_{0}}=\frac{2 \pi n_{0}}{\lambda_{0}}\left(p_{0}, q_{0}, m_{0}\right)$ the Fourier diffraction theorem [4] links the 3D Fourier transform of the scattering potential $f$ with the 2D Fourier transform of the diffracted field $U_{\text {dif }}$ on a given plane at $z=z^{+}$via a mapping on spherical caps whose orientation and position directly depend on the lighting direction $\vec{k}_{0}{ } \propto\left(p_{0}^{j}, q_{0}^{j}, m_{0}^{j}\right)$ : 

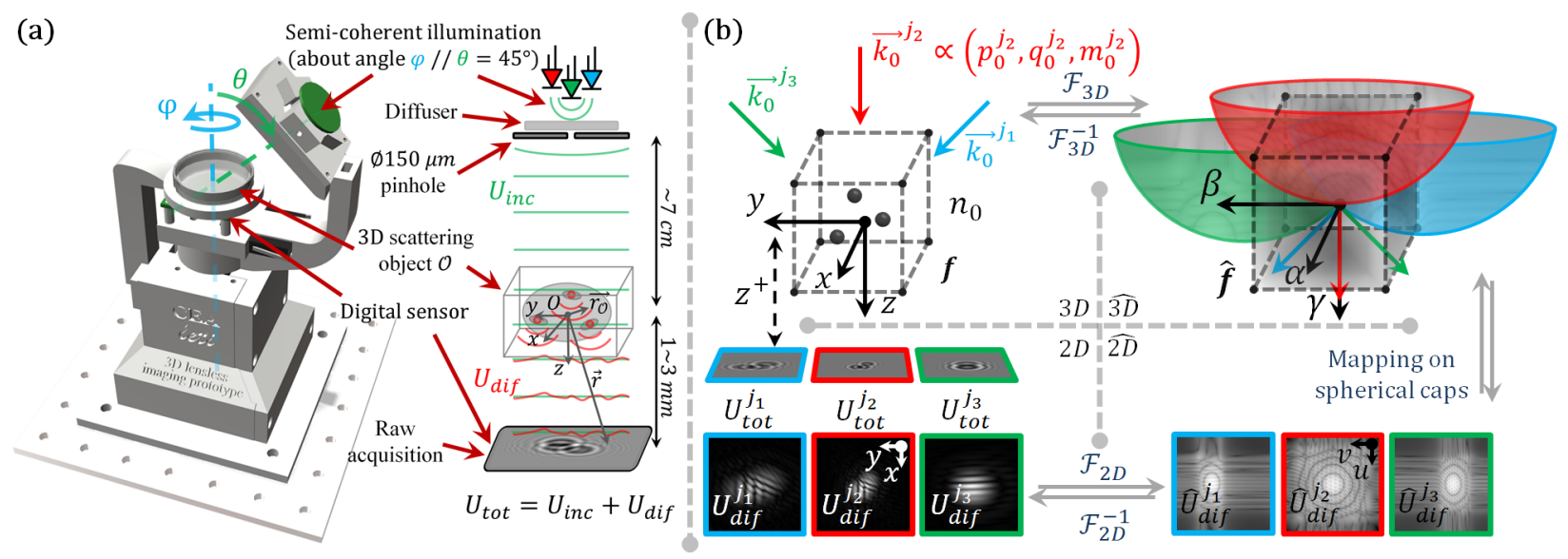

Fig. 1. (a) Experimental setup dedicated to lens-free diffractive tomography and its optical scheme. (b) Illustration of the geometrical interpretation of the Fourier diffraction theorem: the 2D Fourier transforms of the diffracted waves $U_{d i f}^{j}$ are mapped on spherical caps in the 3D frequency domain of the scattering potential $f$. The orientation and radius of these caps directly depend on the illumination directions ${\overrightarrow{k_{0}}}^{j} \propto\left(p_{0}^{j}, q_{0}^{j}, m_{0}^{j}\right)$ and the associated wavelengths $\lambda^{j}$.

$$
\hat{f}(\alpha, \beta, \gamma)=\frac{4 \pi}{i k_{0}^{2}} w e^{-2 i \pi w z^{+}} \hat{U}_{d i f}\left(u, v ; z^{+}\right) \text {with }\left\{\begin{array}{c}
\alpha=u-n_{0} / \lambda \cdot p_{0} \\
\beta=v-n_{0} / \lambda \cdot q_{0} \\
\gamma=w-n_{0} / \lambda . m_{0}
\end{array} \text { and } w(u, v)=\sqrt{\frac{n_{0}^{2}}{\lambda^{2}}-u^{2}-v^{2}}\right.
$$

Two algorithms were implemented to reconstruct the 3D volume (see Fig. 2).

Regularized Gerchberg-Saxton algorithm

Figure 2.a shows the scheme of the Gerchberg-Saxton algorithm [8] which was developed. It presents two subalgorithms.

The first algorithm is a standard Gerchberg-Saxton algorithm in which a constraint is applied on the object [9]. It corresponds to the green arrow in the figure. Nevertheless, the angular coverage of the sample is limited. Moreover the phase of the complex wave is not recorded by the sensor. The algorithm needs a priori information to converge toward an acceptable result. Consequently, a new step is added in the loop, between steps 3 and 4, leading to the blue loop in Fig. 2.a. This step 3.2 consists in regularizing the object $f$. To do so, a minimization problem is defined as:

$$
\tilde{\delta} t=\underset{C(f)}{\operatorname{argmin}} \mu_{L_{1}}\|f\|_{L_{1}, \varepsilon}+\mu_{\nabla}\|\nabla f\|_{L_{1}, \varepsilon}
$$

where $C(f)$ stands for the domain constraints of the solution $\tilde{f},\left(I_{t o t}^{j}\right)_{j \in \llbracket 1, N \rrbracket}$ the $N$ recorded intensities, $\|\cdot\|_{L_{1}}$ the $L_{1}$-norm and $\nabla$ the gradient operator.

The first term is a sparsity prior. The second term is an edge-preserving regularization via a sparsity prior on the gradient of $f$ to enforce smooth and almost uniform volumes while preserving sharp edges. Two hyperparameters $\mu_{L_{1}}$ and $\mu_{\nabla}$ are used to appropriately tune the trade-off between these two regularization terms.

The regularization consists in minimizing the criterion (3), which is performed by a variable metric method with limited memory requirements and bound constraints (VMLMB) algorithm [10], a modified limited memory quasiNewton convex optimization method with Broyden-Fletcher-Goldfarb-Shanno (BFGS) updates and bound constraints.

To accelerate the cleaning of the artefacts, there is no data fidelity term in the cost function (3) which is insured later in the loop by step 4 . As a consequence, the solution of the minimization problem (3) is the volume $f=0$. Thus, a limited number of iterations $n b_{\text {reg }}=10$ is performed to reduce the artifacts while keeping the signal of the object strong enough for the next loop. 
(a)

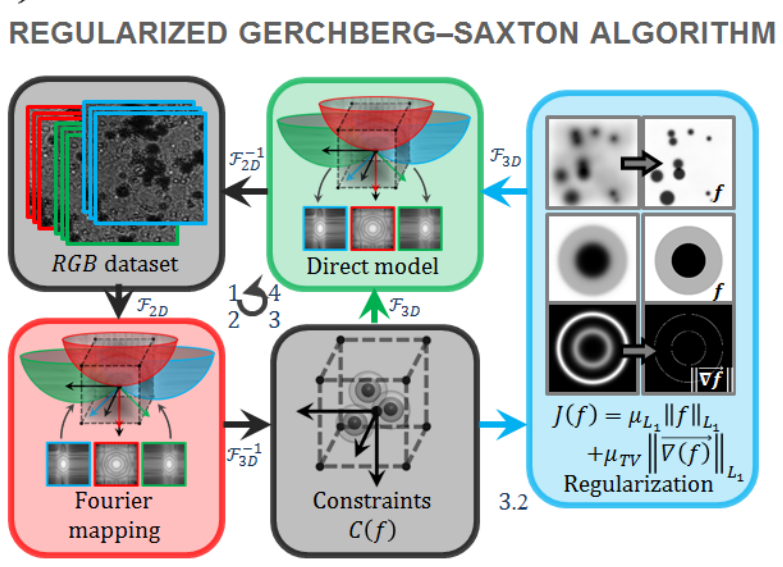

(b)

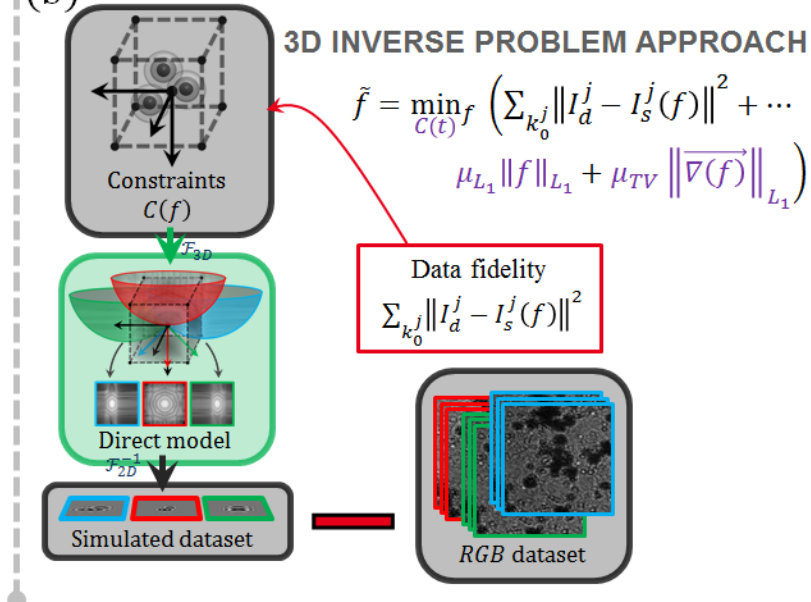

Fig. 2. Scheme of the reconstruction algorithms. (a) Scheme of the Gerchberg-Saxton algorithm. The green arrow corresponds to the standard Gerchberg-Saxton algorithm. To compensate the lack of information of the recorded dataset, a regularization step (the blue arrows) is added. (b) Scheme of the inverse problem approach. Only the direct model is used to simulate a numerical dataset which are compared to the experimental acquisitions.

Inverse problem approach

An inverse problem approach was implemented [11], using the Fourier diffraction theorem (2) as a direct model. As presented on Fig. 2.b, it consists in minimizing the new cost function:

$$
\tilde{f}=\underset{C(f)}{\operatorname{argmin}}\left[\frac{1}{N} \sum_{j=1}^{N}\left\|I_{d}^{j}-I_{s}^{j}(f)\right\|^{2}+\mu_{L_{1}}\|f\|_{L_{1}, \varepsilon}+\mu_{\nabla}\|\nabla f\|_{L_{1}, \varepsilon}\right] \text { with } I_{s}^{j}(f)=\left|U_{i n c}^{j}+U_{d i f}^{j}(f)\right|^{2}
$$

where $C(f)$ stands for the domain constraints of the solution $\tilde{f}$. This minimization problem is solved using the previously mentioned convex optimization algorithm [10].

The method allows to relax the data fidelity terms to take in account the limitations of the model and that the data are noisy and consequently do not have to be perfectly matched by the reconstructions. And as previously, this method appropriately deals with the lack of phase information in the sensor plane and compensates the limited angular coverage, by injecting a priori information on the object to be retrieved via the last two regularization terms.

\section{Results}

The 3D lens-free microscope was tested in incubator on a RWPE1 cell culture which was monitored during two weeks. This cell culture was prepared according to a "no-top-coat" protocol: the cells are directly seeded on the top of a Matrigel ${ }^{\circledR}$ drop, laid down in the Petri dish and can freely move on this 3D surface.

These first results show the potential of this lens-free technology to study 3D cell cultures on large scale in different fields.

\section{D cell motility}

Figure 3.a presents the 3D reconstruction of the cell culture at $t=103 \mathrm{~h} 30 \mathrm{~min}$. The reconstruction parameters are $\varphi \in\left\{0^{\circ} ; 305^{\circ}\right\}, \delta \varphi=9.8^{\circ}, \theta=45^{\circ}, \lambda=R G B, z_{s}=1.66 \mathrm{~mm}, 800 \times 800 \times 128$ voxels of $3.34 \mu \mathrm{m} \times 3.34 \mu \mathrm{m} \times 5.32 \mu \mathrm{m}$ for a final volume: $2.67 \mathrm{~mm} \times 2.37 \mathrm{~mm} \times 0.68 \mathrm{~mm}=4.86 \mathrm{~mm}^{3}$.

This large field of view allows to track cells on long distance. It is emphasized on Fig. 3.b where the maximal values of the temporal stack were kept. The cells displacements leaves tracks on such a visualization. It can be seen that the cells does not follow a random movement but are moving along extended organized trajectories. 

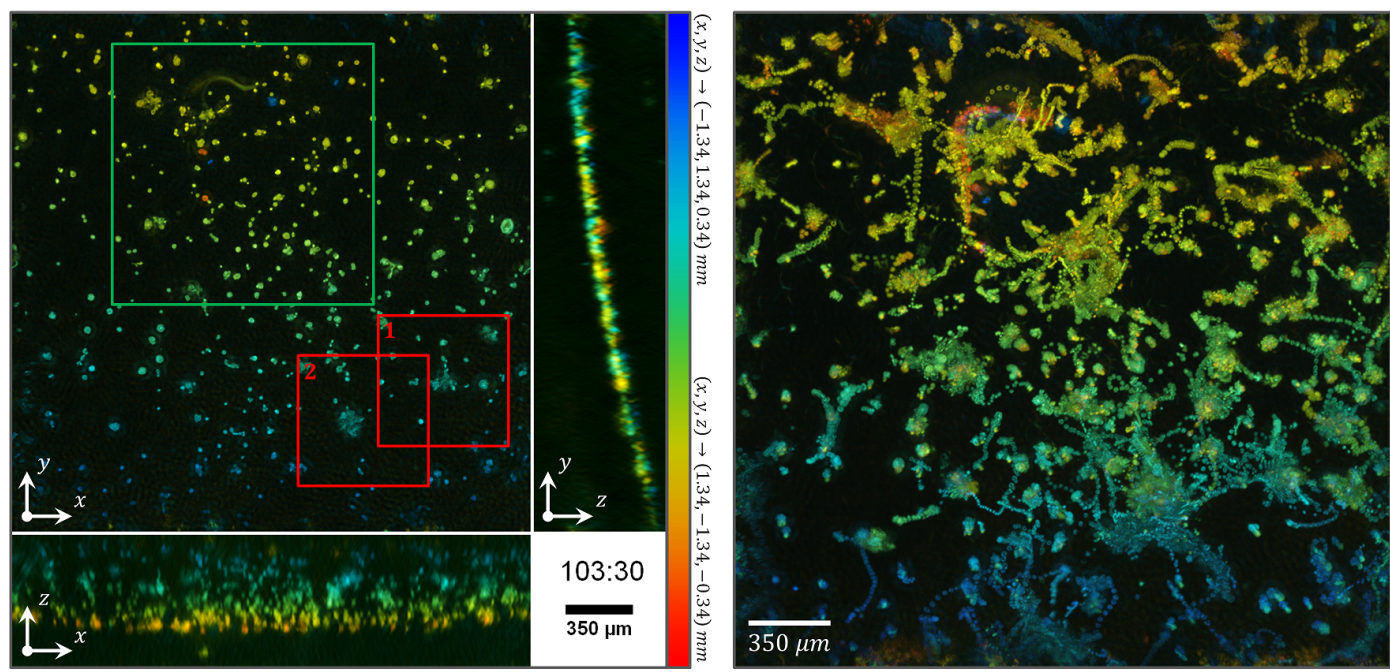

Fig. 3. (a) 3D integrated orthogonal views of the reconstructed volume at $t=103 \mathrm{~h} 30 \mathrm{~min}$. The RWPE1 cell culture was followed during two weeks on the 3D lens-free microscope. The color bar gives the depth in the volume for each views. The blue color encodes for the highest positions ( $z=0.34 \mathrm{~mm}$ for the $x y$-view, $x=-1.34 \mathrm{~mm}$ for the $y z$-view, $y=1.34 \mathrm{~mm}$ for the $x z$-view) and the red color encodes for the deepest positions $(z=-0.34 \mathrm{~mm}$ for the $x y$-view, $x=1.34 \mathrm{~mm}$ for the $y z$-view, $y=-1.34 \mathrm{~mm}$ for the $x z$-view). The green square is the region of interest which was reconstructed at high resolution (see Fig. 5). The red regions of interest emphasize noticeable cell behaviors (see Fig. 4). (b) Maximal value of the temporal stack for each pixel of the $x y$-view. The trajectory of isolated cells can be identified.

Cells-cells interactions

Figure 4 presents two montages extracted from the regions of interest introduced in Fig. 3. These montages emphasize some cells-cells behaviors. Once again, the large field of view of a lens-free microscope allows to find rare phenomena which could not be found neither monitored with classical imaging techniques.

On Fig. 4.a-b, cells are probing each other before merging to shape an organoid. A phenomenon which takes place on more than four days.

On Fig. 4.c-d two organoids are merging into a bigger structure while absorbing other single isolated cells. To to so, they extend cellular branches in the direction of their neighbor. The two branches merge to create one cellular bridge between the organoids which pull on it to merge.

Extracellular matrix-cells interactions

A region of Fig. 3 was selected to be reconstructed at full resolution (the green square). Figure 5.a presents the 3D reconstruction at $t=193 \mathrm{~h} 12 \mathrm{~min}$. The reconstruction parameters are $\varphi \in\left\{0^{\circ} ; 305^{\circ}\right\}, \Delta \varphi=9.8^{\circ}, \theta=45^{\circ}, \lambda=R G B$, $z_{s}=1.66 \mathrm{~mm}, 800 \times 800 \times 200$ voxels of $1.67 \mu \mathrm{m} \times 1.67 \mu \mathrm{m} \times 2.66 \mu \mathrm{m}$ for a final volume: $1.34 \mathrm{~mm} \times 1.34 \mathrm{~mm} \times$ $530 \mu m=1 \mathrm{~mm}^{3}$.

Figure 5.b emphasizes the trajectory of a specific organoid (red arrow). During its movement, it absorbs lots of cell and grow larger. The interesting part is the movement of the surrounding cells which present a back and forth shift. Everything behaves as if the organoid was pulling the surface of the Matrigel ${ }^{\circledR}$ and once it turned, it released the tension. The other cells, just fixed on the Matrigel ${ }^{\circledR}$ follows this movement.

This tension acts more than $300 \mu \mathrm{m}$ from the organoid. This shows the potential of the lens-free microscope to study large scale interaction of cells with their 3D surrounding medium. 

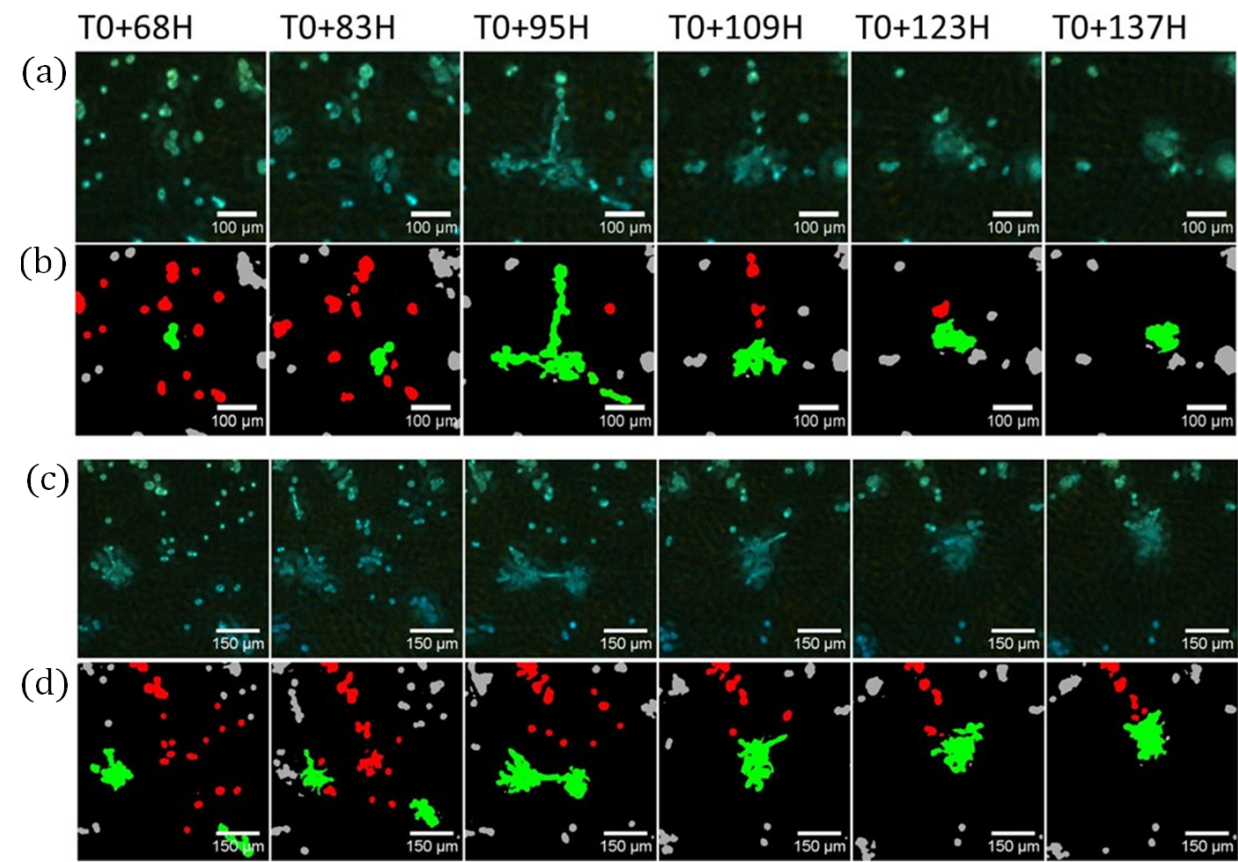

Fig. 4. (a-b) Montage of a movie cropped from Fig. 3.a according to the red region of interest 1. (a) 3D reconstructions at different times. (b) Segmentation of (a): the organoid (green) pulls cells (red) before their merging. (c-d) Montage of a movie cropped from Fig. 3.a according to the red region of interest 2. (c) 3D reconstructions at different times. (d) Segmentation of (c): the two organoids (green) exchanges cells before creating a branch and merging. Some other cells (red) are then pulled before their merging.
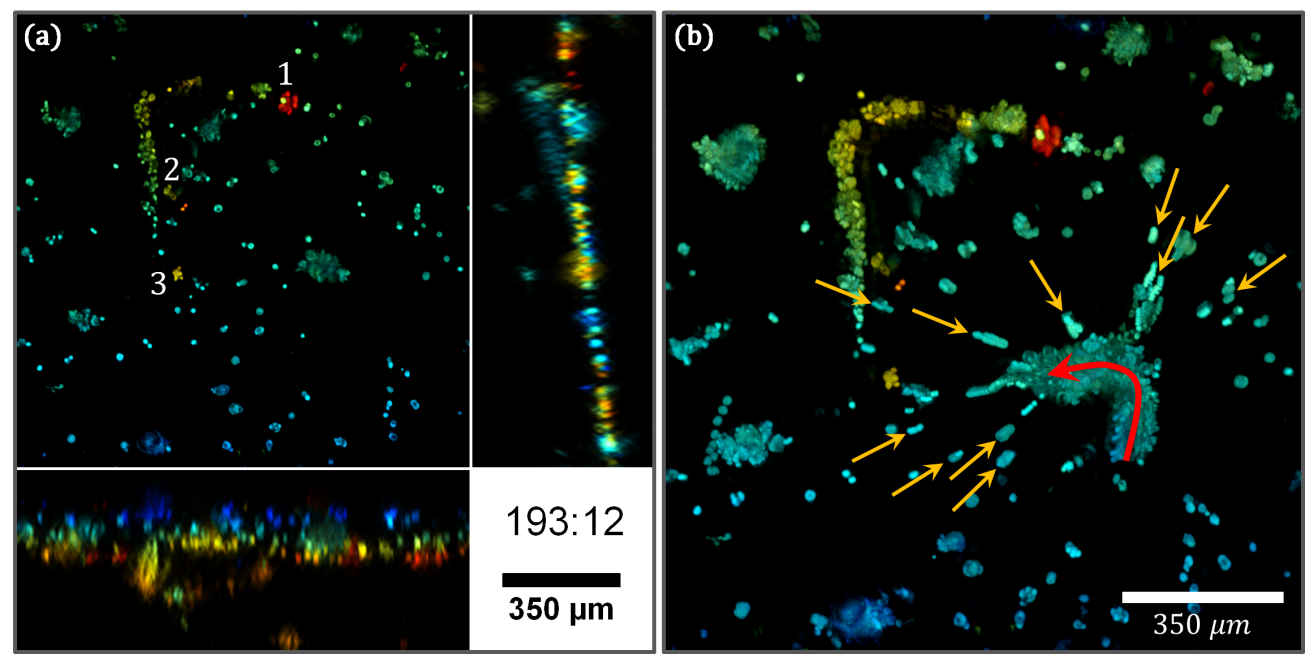

Fig. 5. (a) Reconstruction of the region of interest highlighted in Fig. 3 at $t=193 \mathrm{~h} 12 \mathrm{~min}$ in full resolution. The orthogonal views use the convention introduced in Fig. 3. Three organoids which grew inside the Matrigel ${ }^{\circledR}$ are numbered. See Fig. 6. (b) Maximal value of the temporal stack for each pixel of the $x y$-view between $t=176 \mathrm{~h} 39 \mathrm{~min}$ and $t=208 \mathrm{~h} 23 \mathrm{~min}$. The yellow arrows point at cells fixed in the Matrigel ${ }^{\circledR}$ which highlight its deformation as the organoid moves along the red arrow. 
Developmental biology

On Fig. 5.a, three organoids are highlighted with numbers. They grew inside the Matrigel ${ }^{\circledR}$ matrix. It was consequently possible to follow their development along the two weeks monitoring.
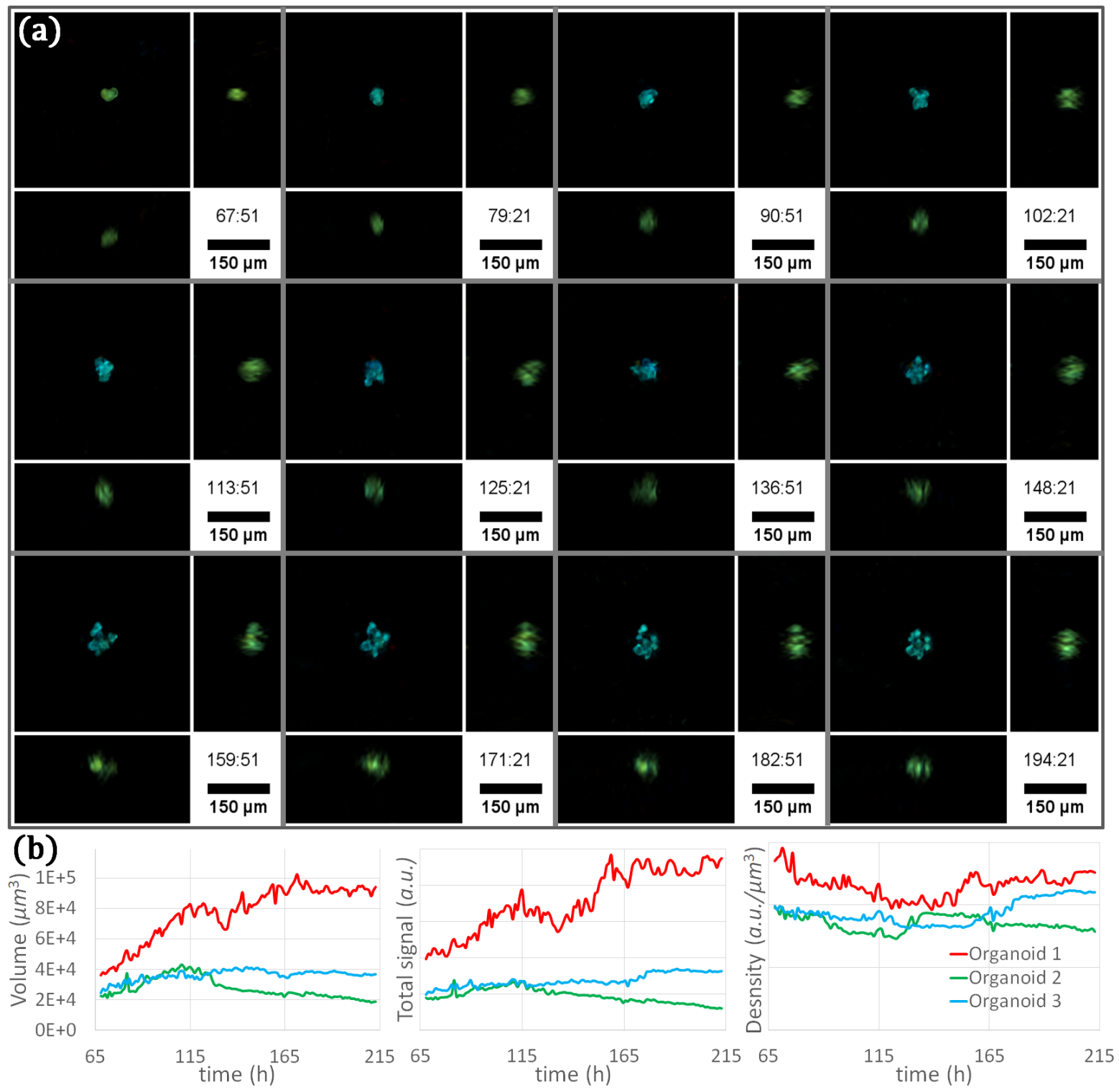

Fig. 6. (a) Montage of the organoid 1 highlighted in Fig. 5. It mainly spreads on two dimensions. (b) The volume of the three organoids is plotted along the time as well as the signal integrated on the organoids. This two quantities give access to the overall density of the organoids (third curve).

Figure 6.a show a montage of the time-lapse done on the organoid 1. The reconstruction parameters are $\varphi \in$ $\left\{0^{\circ} ; 305^{\circ}\right\}, \Delta \varphi=9.8^{\circ}, \theta=45^{\circ}, \lambda=R G B, z_{s}=1.4 \mathrm{~mm}, 256 \times 256 \times 128$ voxels of $1.67^{3} \mu \mathrm{m}^{3}$ for a final volume of $430 \mu \mathrm{m} \times 430 \mu \mathrm{m} \times 210 \mu \mathrm{m}$. One can see that the organoid extends by ejecting some cells on its side.

It is also possible to monitor their volume (see Fig. 6.b). It appears that they grow before reaching a plateau and even retracting for the organoid 2. Even if the reconstruction methods are not proven to be quantitative, it was interesting to draw the overall reconstructed signal on the volume as well as the organoids density (the integrated signal divided by the volume). Their density remains overall constant. 


\section{Results}

In this work we proved the feasibility to adapt a 3D tomographic lens-free microscope to incubator conditions. We were able to monitor a 3D cell culture over an extended period of time without killing the cells.

Moreover, we highlighted that this kind of microscope can pave the way to new kind of experiments on large scale systems in the fields of 3D cell motility, 3D cells-cells interaction, developmental biology as well as the characterization of the 3D extracellular matrix and its interactions with the cell culture.

\section{References}

1. S. V. Kesavan \& al., "High-throughput monitoring of major cell functions by means of lensfree video microscopy," Scientific Reports 4 (2014).

2. I. Pushkarsky, Y. Liu, W. Weaver, T. W. Su, O. Mudanyali, A. Ozcan, and D. Di Carlo, "Automated single-cell motility analysis on a chip using lensfree microscopy," Scientific Reports 4 (2014).

3. Z. Zheng, S. A. Lee, Y. Antebi, M. B. Elowitz, and C. Yang, "The ePetri dish, an on-chip cell imaging platform based on subpixel perspective sweeping microscopy (SPSM)," Proceedings of the National Academy of Sciences, U.S.A 108, 16,889-16,894 (2011).

4. E. Wolf, "Three-dimensional structure determination of semi-transparent objects from holographic data," Optics Communications 1, 153-156 (1969).

5. O. Haeberlé \& al., "Tomographic diffractive microscopy: basics, techniques and perspectives," Journal of Modern Optics 57, 686-699 (2010).

6. F. Momey \& al., "Lensfree diffractive tomography for the imaging of 3D cell cultures," Biomedical Optics Express 7, 949-962 (2016).

7. D. Gabor, "A new microscopic principle," Nature 161, 777778 (1948).

8. R. W. Gerchberg and W. O. Saxton, "A practical algorithm for the determination of the phase from image and diffraction plane pictures," Optik 35, 237-246 (1972).

9. Y. Sung, W. Choi, C. Fang-Yen, K. Badizadegan, R. R. Dasari, and M. S. Feld, "Optical diffraction tomography for high resolution live cell imaging," Optics Express 17, 266-277 (2009).

10. J. Nocedal, "Updating quasi-newton matrices with limited storage," Mathematics of Computation 35, 773-782 (1980).

11. A. Berdeu, F. Momey, B. Laperrousaz, T. Bordy, X. Gidrol, J.-M. Dinten, N. Picollet-D'hahan, and C. Allier, "Comparative study of fully three-dimensional reconstruction algorithms for lens-free microscopy," Appl. Opt. 56, 3939-3951 (2017). 